\title{
Oral Nucleotides Only Minimally Improve 5-Fluorouracil-Induced Mucositis in Rats
}

Suzanne Mashtoub, ${ }^{1,2}$, Benjamin Feo ${ }^{3}$, Alexandra L Whittaker ${ }^{3}$, Kerry A Lymn ${ }^{3,4}$, Daniel Martinez-Puig ${ }^{5}$, Gordon S Howarth ${ }^{2,3 *}$

${ }^{1}$ School of Medicine and Pharmacology, The University of Western Australia, Fremantle Hospital, Fremantle, Western Australia 6160

${ }^{2}$ Department of Gastroenterology, Women's and Children’s Hospital, North Adelaide, South Australia 5006

${ }^{3}$ School of Animal and Veterinary Sciences, The University of Adelaide, Roseworthy Campus, South Australia 5371

${ }^{4}$ Women’s and Children’s Health Research Institute, North Adelaide, South Australia 5006 ${ }^{5}$ Bioiberica S.A., Barcelona, Spain

*Corresponding author: Professor Gordon S Howarth, School of Animal and Veterinary Sciences, The University of Adelaide, Roseworthy Campus, South Australia, Australia, 5371 Phone: 618-8313-7885 Fax: 618-8313-7972 Email: gordon.howarth@adelaide.edu.au

Running Title: Oral nucleotides and experimental mucositis

Keywords: Nucleotides; Nucleoforce; Yeast; Chemotherapy; Mucositis; Rat 


\section{ABSTRACT}

Background. Chemotherapy-induced mucositis is characterized by inflammation and ulceration of the intestinal mucosa, compromising intestinal function. Exogenous nucleotides have been reported to repair the mucosa. The nucleotide preparation, Nucleoforce F0328 (Nucleoforce), was investigated for its potential to ameliorate intestinal mucositis in rats. Methods. Female Dark Agouti rats (n=8/group) were gavaged once daily with Nucleoforce (175mg/kg) or water from days 0-8 and injected (i.p.) with 5-Fluorouracil (5-FU:150mg/kg) or saline on day 5. Histological parameters (disease severity, crypt depth and villus height measurements) and myeloperoxidase activity were quantified. $\mathrm{p}<0.05$ was considered significant. Results. Jejunal and ileal histological disease severity scores were significantly increased by 5-FU, compared to normal controls $(\mathrm{p}<0.05)$. Nucleoforce treatment in 5 -FUinjected rats significantly reduced jejunal and ileal disease severity compared to 5-FU controls $(\mathrm{p}<0.05)$. In 5-FU-injected rats, jejunal and ileal villus heights and crypt depths were significantly decreased compared to 5-FU controls, with no additional Nucleoforce effect ( $>>0.05$ ). Intestinal myeloperoxidase activity was significantly elevated by 5-FU (8.8-fold), compared to normal controls $(\mathrm{p}<0.05)$, which was not normalised by Nucleoforce treatment ( $>0.05$ ). Conclusions. Nucleoforce only partially improved parameters associated with experimentally-induced mucositis. Future studies could investigate increased concentrations, more frequent administration, or protective microencapsulation delivery methods, to increase bioavailability. 


\section{INTRODUCTION}

The anti-metabolite chemotherapeutic agent, 5-Fluorouracil (5-FU), is commonly used to treat a variety of malignancies including breast and bowel cancer ${ }^{1,2}$. However, its nonspecific mechanism of action results in collateral damage to enterocytes lining the small intestine and the subsequent manifestation of a condition known as mucositis. Mucositis is characterised by severe pain, nausea, bloating, diarrhoea and loss of appetite, accompanied by histological features of decreased villus length and crypt disruption ${ }^{3}$.

Mucositis affects up to 500,000 cancer sufferers each year ${ }^{4}$ and severely reduces quality of life in patients undergoing chemotherapy regimens ${ }^{5}$. Mucositis can affect the entire gastrointestinal tract, but most commonly affects the mucosa of the mouth and small intestine. Chemotherapy with agents such as 5-FU induces enterocyte apoptosis combined with decreased gut barrier function, allowing translocation of pathogenic bacteria and endotoxins into the bloodstream. This can result in systemic infection and sometimes death. Mucositis can be so severe that chemotherapy regimens must be restructured, or in some cases discontinued, altering patient prognosis ${ }^{5}$.

There are currently no definitive treatments for chemotherapy-induced mucositis, although agents have been identified that are able to combat specific features of mucositis pathogenesis. Although reduced mucositis severity has been reported following treatment with keratinocyte growth factor $1^{6-8}$, few other agents have progressed beyond the experimental stage. Recently, a somewhat disparate group of naturally-sourced agents have demonstrated improvements in mucositis symptomatology in the experimental setting. These include a whey-derived growth factor extract ${ }^{9}$, plant extracts such as Grape Seed Extract ${ }^{10,11}$ and Iberogast ${ }^{12}$, animal-sourced oils such as Emu Oil ${ }^{13-16}$ and Lyprinol ${ }^{17}$ and certain 
probiotics $^{18}$. However, despite the effectiveness of these compounds, the need to pursue alternative treatment modalities still remains.

Purine and pyrimidine nucleotides are vital in structural, metabolic, energetic and regulatory processes and are particularly important in rapidly-dividing cells such as intestinal enterocytes ${ }^{19-21}$ where they are essential for proliferation and in the repair process ${ }^{19}$. Endogenous supply of nucleotides is maintained through de novo synthesis and the salvage pathway $^{21}$. Cells lining the intestinal epithelium lack significant capacity for de novo synthesis of such nucleotides and require an exogenous source of purine and pyrimidine bases $^{20,22-24}$. Several studies have described the ability of exogenous nucleotides to either ameliorate intestinal damage or to promote repair ${ }^{21,25,26}$. However, specific effects on chemotherapy-induced mucositis have not yet been described.

Accordingly, the present study investigated an orally administered, yeast-derived nucleotide preparation termed Nucleoforce F0328, for its potential to ameliorate 5-FU-induced intestinal mucositis in rats. 


\section{METHODS}

\section{Nucleoforce}

Nucleoforce F0328, a nucleotide preparation derived from yeast, was provided by Bioiberica S.A., Barcelona, Spain. It is a beige powder that is readily soluble in water (15\%). Nucleoforce was prepared as an aqueous stock solution $(26.25 \mathrm{mg} / \mathrm{ml})$ and aliquoted into individual Eppendorf tubes (1.5ml) before storage at -80C.

\section{Animal Study}

Female Dark Agouti rats $(\mathrm{n}=32 ; 110-150 \mathrm{~g}$, Laboratory Animal Services, University of Adelaide, Adelaide, South Australia) were housed in individual metabolism cages (Tecniplast, PA, USA) in a light and climate controlled room. Rats were placed in metabolism cages 2 days prior to commencement of the experimental trial, to allow for acclimatisation. Rats were given ad libitum access to water and food (18\% casein-based diet $)^{27}$ and were randomly assigned to one of four treatment groups ( $n=8$ per group): a negative control of Saline and Water; Saline and Nucleoforce; 5-FU (Mayne Pharma Pty Ltd, Victoria, Australia) and water; or 5-FU and Nucleoforce. Rats were gavaged with Nucleoforce $(175 \mathrm{mg} / \mathrm{kg})$ or water from days 0 to day 8 . On day 5 , rats were intraperitoneally injected with either saline (control group) or 5-FU (150 mg/kg). All experimentation adhered to the Australian Code of Practice for the Care and Use of Animals for Scientific Purposes and was approved by the Animal Ethics Committee of The University of Adelaide.

\section{Daily Metabolic Data and Disease Activity Index}

Body weight, food and water intake, and fecal and urine output were monitored and measured daily. The severity of mucositis was assessed daily, using a disease activity index (DAI), which scored body weight loss, rectal bleeding, stool consistency, and overall general 
condition of the animal, increasing in severity on a scale of $0-3$ for each parameter, which was totalled to achieve an overall DAI, as described previously ${ }^{28,29}$. Overall condition was determined by (1) mobility/agility: healthy rats were considered quite active, whereas rats affected by 5-FU characteristically became very weak and feeble, sitting hunched with very little movement and (2) fur: healthy rats were well-groomed with fur flat to the body, whereas 5-FU-injected rats became scruffy in appearance, with ruffled fur.

\section{Sucrose Breath Test}

The sucrose breath test (SBT) was performed on day 8 immediately prior to sacrifice as a non-invasive measure of small intestinal brush-border disaccharidase activity ${ }^{30}$. Following an overnight fast, rats were gavaged with $1 \mathrm{ml}$ of an aqueous sucrose solution $(0.25 \mathrm{~g} / \mathrm{ml})$ labelled with the non-radioactive isotope ${ }^{13} \mathrm{C}$. Rats were then placed in sealed plastic containers for 2 minutes, at which time a $10 \mathrm{ml}$ sample of breath was collected into an evacuated tube. Breath samples were collected at $0,15,30,45,60,75,90,105$ and 120 minutes post-sucrose solution gavage. Samples were analysed for ${ }^{13} \mathrm{C}$ content by isotope ratio mass spectrometry, and the results expressed as mean percentage cumulative dose (\%CD) at 75, 90, 105 and 120 minutes ${ }^{30}$.

\section{Tissue collection}

Following sacrifice by $\mathrm{CO}_{2}$ asphyxiation and cervical dislocation on day 8 , the gastrointestinal tract of each animal was removed, measured, emptied of contents and weighed. Following measurements, 2cm segments of small intestinal tract (jejunum, jejunumileum junction (JI) and ileum) were collected and placed in $10 \%$ buffered formalin for histological analysis. Subsequently, segments $(4 \mathrm{~cm})$ directly adjacent to the corresponding histological samples were snap-frozen in liquid nitrogen for biochemical analysis. Samples 
were stored at $-80^{\circ} \mathrm{C}$ until prepared for analysis by homogenization in $10 \mathrm{mM}$ phosphate buffer. The visceral organs (thymus, heart, spleen, liver, kidneys and lungs) were removed, weighed and discarded.

\section{Histological Analyses}

Tissue samples were transferred from $10 \%$ buffered formalin into a $70 \%$ ethanol solution 24 hours post-collection. Specimens were then routinely processed and embedded in paraffin wax and $4 \mu \mathrm{m}$ sections were prepared and stained with haematoxylin and eosin (H\&E). The overall histological damage severity was assessed by a semi-quantitative scoring system, as per Howarth et al. ${ }^{9}$ in a blinded fashion, using an Olympus CX-13 light microscope (Olympus, Tokyo, Japan). The parameters assessed were: Villus/crypt ratio, enterocyte disruption, reduction in goblet cell numbers, crypt disruption, polymorphonuclear cell infiltration, oedema of the submucosa and thickening of the muscularis externa. Each parameter was allocated a score between 0 (no damage) to 3 (extreme damage). Small intestinal villus height and crypt depth were measured in the jejunum and ileum (40 villi and 40 crypts per section) using a light microscope (Olympus BH-2, Tokyo, Japan) and digital camera (Sony, Tokyo, Japan) using analysis imaging software (Version 5.1, Olympus, Tokyo, Japan) $)^{9}$.

\section{Myeloperoxidase (MPO) activity}

Myeloperoxidase levels in the small intestine were determined as an indicator of neutrophil infiltration, and hence, acute inflammation, using techniques described by Howarth et al. ${ }^{9}$ Briefly, jejunal tissue samples were centrifuged at $13000 \mathrm{~g}$ for $10 \mathrm{~min}$, after which the supernatant was discarded and the tissue homogenate re-suspended in $0.5 \%$ hexadecyltrimethyl ammonium bromide (HTAB). After vortexing for 2 min, samples were 
re-centrifuged, at $5000 \mathrm{~g}$ for $2 \mathrm{~min}$. The supernatants were then aliquoted into 96 well plates. After the addition of o-dianisidine reagent, the change in absorbance was measured at $450 \mathrm{~nm}$ on an ELISA plate reader (Sunrise Microplate Absorbance Reader, Tecan Austria GmbH, Grödig, Austria).

\section{Statistical Analyses}

Statistical analyses were performed with SPSS (Version 15, Chicago, USA). Daily metabolic data were analysed and compared both within and between groups using an ANOVA with a Holme’s post hoc test. Histological severity scores were analysed with a Kruskal Wallis test and a Bonferroni post hoc test and expressed as median (range). The remaining data were analysed by a one-way ANOVA with a Tukey post hoc test and expressed as mean \pm standard error of the mean (SEM). $\mathrm{p}<0.05$ was considered significant. 


\section{RESULTS}

\section{Metabolic data}

Prior to administration of 5-FU or saline, body weight increased in all animals from days 0-4 $(\mathrm{p}<0.05)$, with no significant differences between groups ( $>0.05$; Figure 1$)$. Rats injected with 5-FU displayed significantly less bodyweight on days 6-8, compared with normal controls, including those treated with Nucleoforce $(\mathrm{p}<0.001$; Figure 1$)$. Rats were fasted on days 0,5 and 8 for the sucrose breath test, accounting for the observed weight loss (Figure 1).

In normal rats, Nucleoforce did not significantly affect metabolic parameters compared to normal controls ( $>>0.05$; Table 1). Food intake and faecal output were significantly decreased by 5 -FU injection, compared with normal controls $(\mathrm{p}<0.05$; Table 1$)$, whilst water intake and urine output remained unchanged. Nucleoforce did not improve food intake and faecal output in 5-FU-injected rats compared to 5-FU controls ( $\mathrm{p}>0.05$; Table 1).

\section{Sucrose breath test}

Brush border sucrase activity was used as an indicator of the health and maturity of epithelial cells which line the small intestine, assessed using the SBT. On day 8, 72 hours post-5-FU injection, the percentage cumulative dose of ${ }^{13} \mathrm{C}(\% \mathrm{CD})$ at 75, 90, 105 and 120 minutes remained unchanged following Nucleoforce treatment in normal rats compared to normal controls ( $>0.05$; Figure 2). In 5-FU-injected rats, \%CD was significantly decreased compared to normal controls ( $<<0.001$; Figure 2). \%CD was not improved by Nucleoforce in 5-FU-injected rats compared to 5-FU controls (Figure 2; $\mathrm{p}>0.05$ ). 


\section{Visceral organ weights}

Organ weights were expressed as a proportion of body weight. Heart, liver, spleen, thymus, lungs, right kidney and left kidney weights were not significantly affected by Nucleoforce treatment in normal rats compared to normal controls ( $\mathrm{p}>0.05$; Table 2). Spleen weight was significantly decreased by $5-\mathrm{FU}$ administration compared to normal controls $(\mathrm{p}<0.05)$. However, 5-FU did not significantly alter other visceral organ weights. Nucleoforce treatment in 5-FU-injected rats resulted in significantly decreased spleen weights compared to 5-FU controls $(\mathrm{p}<0.05$; Table 2$)$.

\section{Intestinal organ weights and lengths}

In normal rats, Nucleoforce did not significantly affect intestinal organ weights or lengths compared to normal controls ( $\mathrm{p}>0.05$; Table 3). 5-FU significantly decreased jejuno-ileum weights compared to normal controls ( $<<0.001$; Table 3 ). Neither 5 -FU nor Nucleoforce affected other intestinal organ weights or lengths ( $\mathrm{p}>0.05$; Table 3 ).

\section{Histological severity scoring}

In normal rats, Nucleoforce treatment did not significantly affect histologically-assessed severity scores in the jejunum ( $>0.05$ ), however, Nucleoforce increased severity scores in the ileum compared to normal controls ( $\mathrm{p}=0.043$; Table 4). 5-FU administration significantly increased severity scores in both the jejunum and ileum compared to normal controls $(\mathrm{p}<0.05)$. Importantly, however, Nucleoforce treatment significantly decreased severity scores in the jejunum $(\mathrm{p}=0.039)$ and ileum $(\mathrm{p}=0.041)$ of 5 -FU-injected rats compared to 5 -FU controls (Table 4). 


\section{Small intestinal histology}

Jejunal villus height and crypt depth were not significantly affected by Nucleoforce treatment in normal rats compared to normal controls ( $>>0.05$; Figure 3a). However, in the ileum, Nucleoforce treatment significantly decreased villus height $(\mathrm{p}<0.001)$, with no effect on crypt depth ( $>0.05$ ), compared to normal controls (Figure 3b). 5-FU administration significantly decreased villus height and crypt depth in the jejunum (villus height, $\mathrm{p}<0.001$; crypt depth, $\mathrm{p}<0.05$; Figure $3 \mathrm{a})$ and ileum $(\mathrm{p}<0.001$; Figure $3 \mathrm{~b})$ compared to normal controls. In 5 -FUinjected rats, Nucleoforce treatment failed to normalise villus height and crypt depth measurements compared to 5-FU controls ( $\mathrm{p}>0.05)$.

\section{Myeloperoxidase (MPO) activity}

In normal rats, Nucleoforce did not significantly affect jejunal MPO activity compared to normal controls (Figure 4; $>0.05$ ). MPO activity was significantly increased in the jejunum of 5-FU-injected rats compared to normal controls ( $\mathrm{p}<0.001$; Figure 4). In 5-FU-injected rats, Nucleoforce treatment did not significantly impact MPO activity compared to 5-FU controls ( $<<0.05$; Figure 4). 


\section{DISCUSSION}

The present study determined the effects of the nucleotide preparation known as Nucleoforce F0328 on a rat model of chemotherapy-induced mucositis. Nucleoforce, at a daily bolus dose of $175 \mathrm{mg} / \mathrm{kg}$, only minimally improved selected indicators of mucositis, extending primarily to an improvement in food intake with a partial attenuation of body weight loss associated with chemotherapy. Nevertheless, Nucleoforce reduced the severity of small intestinal damage as measured by the histological severity score in both the jejunum and ileum. However, other parameters associated with 5-FU induced mucositis were not significantly affected by Nucleoforce.

In the current study, Nucleoforce primarily decreased injury in the proximal small intestine, as measured by the histological severity score, although there were no significant effects on villus height and crypt depth.

Although generally minimal in degree, the effects of Nucleoforce on most indicators of mucositis pathogenesis in the current study may not necessarily have indicated an absence of efficacy. Gastric degradation of nucleotides prior to reaching the small intestine could represent an alternative explanation. A study by Belo et al. (2006) demonstrated the ability of nucleotides to decrease indomethacin-induced gastric damage in rats, highlighting the possibility of reduced bioavailability of nucleotides in the small intestine as opposed to the stomach $^{26}$. Although nucleotides are known to be involved in maturation of the small intestinal epithelium, and hence brush border enzyme expression ${ }^{31}$, the absence of any Nucleoforce effect on sucrase activity, as determined by the sucrose breath test, may have been a further result of insufficient bioactivity in the proximal intestine. Future studies could consider an intravenous administration route to minimise nucleotide degradation in the small intestine $^{23}$. A second explanation for the minimal efficacy of Nucleoforce could have 
represented its temporal application in relation to the time of intestinal injury. In the study by Belo et al. (2006), rats were sacrificed only three hours post-indomethacin treatment, whereas in the current study, rats were killed 72 hours after 5 -FU induced injury ${ }^{26}$. It is therefore possible that Nucleoforce may have demonstrated therapeutic benefit on the indicators of mucositis in the immediate period following 5-FU administration. More comprehensive timecourse studies should be considered in future studies, especially at earlier time-points post 5FU.

Following administration of 5-FU, spleen weights were significantly decreased and liver weight significantly increased, consistent with previous findings in studies employing this model of mucositis ${ }^{32,33}$. However, no additional effects of Nucleoforce were apparent in either normal or chemotherapy-injected rats. Considered together, this contributes to the safety information for Nucleoforce as it revealed that Nucleoforce was not contributing to hepatotoxicity nor to the aberrant stimulation of the systemic immune response.

In conclusion, Nucleoforce only minimally improved selected parameters of small intestinal mucositis. Although initial indications for Nucleoforce therapy in mucositis are not encouraging, future studies should consider strategies to maintain nucleotide bioactivity and bioavailability by either increasing dose, frequency of administration, or alternatively, the use of targeted release microencapsulation systems. 


\section{ACKNOWLEDGEMENTS}

This work was funded by Bioiberica SA, Barcelona, Spain in collaboration with Nidor Pty Ltd, Melbourne, Victoria, Australia. Dr Suzanne Mashtoub is the recipient of the National Health and Medical Research Council Postdoctoral (Peter Doherty) Australian Biomedical Fellowship. Professor Gordon S Howarth is supported by a Senior Research Fellowship from the South Australian Health and Medical Research Institute and Cancer Council of South Australia. 


\section{REFERENCES}

1. Stringer AM, Gibson RJ, Logan RM, Bowen JM, Yeoh AS, et al. Irinotecan-induced mucositis is associated with changes in intestinal mucins. Cancer Chemother Pharmacol; 64:123-32 2009.

2. Whitford EJ, Cummins AG, Butler RN, Prisciandaro LD, Fauser JK, et al. Effects of Streptococcus thermophilus TH-4 on intestinal mucositis induced by the chemotherapeutic agent, 5-Fluorouracil (5-FU). Cancer Biol Ther; 8:505-11 2009.

3. Lalla RV, Peterson DE. Treatment of mucositis, including new medications. Cancer J; 12:348-54 2006.

4. Duncan M, Grant G. Oral and intestinal mucositis - causes and possible treatments. Aliment Pharmacol Ther; 18:853-74 2003.

5. Sonis ST. The pathobiology of mucositis. Nat Rev Cancer; 4:277-84 2004.

6. Blijlevens N, Sonis S. Palifermin (recombinant keratinocyte growth factor-1): a pleiotropic growth factor with multiple biological activities in preventing chemotherapy- and radiotherapy-induced mucositis. Ann Oncol; 18:817-26 2007.

7. Sonis ST. Efficacy of palifermin (keratinocyte growth factor-1) in the amelioration of oral mucositis. Core Evid; 4:199-205 2010.

8. Yazbeck R, Howarth GS, Borges L, Geier MS, Smith CL, et al. Non-invasive detection of a palifermin-mediated adaptive response following chemotherapy-induced damage to the distal small intestine of rats. Cancer Biol Ther; 12:399-406 2011.

9. Howarth GS, Francis GL, Cool JC, Xu X, Byard RW, et al. Milk growth factors enriched from cheese whey ameliorate intestinal damage by methotrexate when administered orally to rats. J Nutr; 126:2519-30 1996.

10. Cheah KY, Howarth GS, Yazbeck R, Wright TH, Whitford EJ, et al. Grape seed extract protects IEC-6 cells from chemotherapy-induced cytotoxicity and improves 
parameters of small intestinal mucositis in rats with experimentally-induced mucositis. Cancer Biol Ther; 8:382-90 2009.

11. Cheah KY, Bastian SE, Acott TM, Abimosleh SM, Lymn KA, et al. Grape seed extract reduces the severity of selected disease markers in the proximal colon of dextran sulphate sodium-induced colitis in rats. Dig Dis Sci; 58:970-7 2013.

12. Wright TH, Yazbeck R, Lymn KA, Whitford EJ, Cheah KY, et al. The herbal extract, Iberogast, improves jejunal integrity in rats with 5-Fluorouracil (5-FU)-induced mucositis. Cancer Biol Ther; 8:923-9 2009.

13. Abimosleh SM, Lindsay RJ, Butler RN, Cummins AG, Howarth GS. Emu oil increases colonic crypt depth in a rat model of ulcerative colitis. Dig Dis Sci; 57:887-96 2012.

14. Abimosleh SM, Tran CD, Howarth GS. Emu Oil: a novel therapeutic for disorders of the gastrointestinal tract? J Gastroenterol Hepatol; 27:857-61 2012.

15. Mashtoub S, Tran CD, Howarth GS. Emu oil expedites small intestinal repair following 5-fluorouracil-induced mucositis in rats. Experimental biology and medicine; 238:1305-17 2013.

16. Abimosleh SM, Tran CD, Howarth GS. Emu oil reduces small intestinal inflammation in the absence of clinical improvement in a rat model of indomethacin-induced enteropathy. Evid Based Complement Alternat Med; 2013:429706 2013.

17. Torres DM, Tooley KL, Butler RN, Smith CL, Geier MS, et al. Lyprinol only partially improves indicators of small intestinal integrity in a rat model of 5-fluorouracil-induced mucositis. Cancer Biol Ther; 7:295-302 2008.

18. Tooley KL, Howarth GS, Lymn KA, Lawrence A, Butler RN. Oral ingestion of streptococcus thermophilus diminishes severity of small intestinal mucositis in methotrexate treated rats. Cancer Biol Ther; 5:593-600 2006. 
19. Nunez MC, Ayudarte MV, Morales D, Suarez MD, Gil A. Effect of dietary nucleotides on intestinal repair in rats with experimental chronic diarrhea. JPEN J Parenter Enteral Nutr; 14:598-604 1990.

20. Veerabagu MP, Meguid MM, Oler A, Levine RA. Intravenous nucleosides and a nucleotide promote healing of small bowel ulcers in experimental enterocolitis. Dig Dis Sci; 41:1452-7 1996.

21. Carver JD. Dietary nucleotides: cellular immune, intestinal and hepatic system effects. J Nutr; 124:144S-8S 1994.

22. Uauy R, Quan R, Gil A. Role of nucleotides in intestinal development and repair: implications for infant nutrition. J Nutr; 124:1436S-41S 1994.

23. Sukumar P, Loo A, Magur E, Nandi J, Oler A, et al. Dietary supplementation of nucleotides and arginine promotes healing of small bowel ulcers in experimental ulcerative ileitis. Dig Dis Sci; 42:1530-6 1997.

24. Ono K, Mawatari K, Harada N, Takahashi A, Sakai T, et al. The nucleoside and nucleotide mixture (OG-VI) rescues intestinal-like epithelial cells from the cytotoxicity of chemotherapeutic agents. J Med Invest; 54:235-42 2007.

25. Bueno J, Torres M, Almendros A, Carmona R, Nunez MC, et al. Effect of dietary nucleotides on small intestinal repair after diarrhoea. Histological and ultrastructural changes. Gut; 35:926-33 1994.

26. Belo A, Marchbank T, Fitzgerald A, Ghosh S, Playford RJ. Gastroprotective effects of oral nucleotide administration. Gut; 55:165-71 2006.

27. Tomas FM, Knowles SE, Owens PC, Read LC, Chandler CS, et al. Effects of fulllength and truncated insulin-like growth factor-I on nitrogen balance and muscle protein metabolism in nitrogen-restricted rats. J Endocrinol; 128:97-105 1991. 
28. Murthy SN, Cooper HS, Shim H, Shah RS, Ibrahim SA, et al. Treatment of dextran sulfate sodium-induced murine colitis by intracolonic cyclosporin. Dig Dis Sci; 38:1722-34 1993.

29. Howarth GS XC, Read LC. Predisposition to colonic dysplasia is unaffected by continuous administration of insulin-like growth factor-I for twenty weeks in a rat model of chronic inflammatory bowel disease. Growth Factors; 18:119-33 2000.

30. Pelton NS, Tivey DR, Howarth GS, Davidson GP, Butler RN. A novel breath test for the non-invasive assessment of small intestinal mucosal injury following methotrexate administration in the rat. Scand J Gastroenterol; 39:1015-6 2004.

31. Ortega MA, Nunez MC, Gil A, Sanchez-Pozo A. Dietary nucleotides accelerate intestinal recovery after food deprivation in old rats. J Nutr; 125:1413-8 1995.

32. Cool JC, Dyer JL, Xian CJ, Butler RN, Geier MS, et al. Pre-treatment with insulin-like growth factor-I partially ameliorates 5-fluorouracil-induced intestinal mucositis in rats. Growth Horm IGF Res; 15:72-82 2005.

33. Mauger CA, Butler RN, Geier MS, Tooley KL, Howarth GS. Probiotic effects on 5fluorouracil-induced mucositis assessed by the sucrose breath test in rats. Dig Dis Sci; 52:612-9 2007. 


\section{FIGURE LEGENDS}

Figure 1. Daily body weight change of rats from days -2 to 8 . Female Dark Agouti rats were gavaged daily with Nucleoforce from days 0-8 and intraperitoneally injected with saline or 5FU (150 mg/kg) on day 5. Rats were fasted on days 0, 5 and 8. Data are expressed as mean (\% of starting bodyweight) \pm SEM. * indicates $\mathrm{p}<0.05$ compared to day 0 ; *** indicates $\mathrm{p}<0.001$ compared to saline + water.

Figure 2. Small intestinal sucrase activity, measured using the ${ }^{13} \mathrm{C}$-sucrose breath test at 75 , 90, 105 and 120 minutes post sucrose gavage on day of kill (day 8). Female Dark Agouti rats were gavaged daily with Nucleoforce from days 0-8 and intraperitoneally injected with saline or 5 -FU $(150 \mathrm{mg} / \mathrm{kg})$ on day 5 . Data are expressed as mean (\% cumulative dose of $\left.{ }^{13} \mathrm{C}\right) \pm$ SEM. *** indicates $\mathrm{p}<0.001$ compared to saline + water.

Figure 3. Villus height and crypt depth measurements in the (a) jejunum and (b) ileum of rats. Female Dark Agouti rats were gavaged daily with Nucleoforce from days 0-8 and intraperitoneally injected with saline or 5-FU (150 mg/kg) on day 5. Data are expressed as mean (length; $\mu \mathrm{m}) \pm$ SEM. ${ }^{* * *}$ indicates $\mathrm{p}<0.001$, * indicates $\mathrm{p}<0.05$ compared to saline + water.

Figure 4. Myeloperoxidase activity indicative of acute inflammation in the rat jejunum following daily gavages of Nucleoforce and intraperitoneal saline or 5-FU injections on day 5. Data are expressed as mean (myeloperoxidase activity; units per milligram; U/mg) \pm SEM. $* * *$ indicates $\mathrm{p}<0.001$ compared to saline + water. 